\title{
Opposing consequences of IL-23 signaling mediated by innate and adaptive cells in chemically induced colitis in mice
}

\author{
JH Cox $^{1}$, NM Kljavin ${ }^{1}$, N Ota ${ }^{2}$, J Leonard ${ }^{1}$, M Roose-Girma ${ }^{1}$, L Diehl $^{3}$, W Ouyang ${ }^{2}$ and N Ghilardi ${ }^{1,2}$
}

The interleukin-23 (IL-23) pathway has emerged as a promising therapeutic target for inflammatory bowel disease. Although the pathogenic role of IL-23 receptor (IL-23R) on T lymphocytes is well established, its function on innate immune cells has not been thoroughly examined. Here we investigate the consequence of IL-23R deletion in dextran sulfate sodium (DSS)-induced colitis. In IL23R ${ }^{-/-}$and IL23p19-/- mice, we observed decreased weight loss and reduced leukocyte infiltrate following DSS exposure. Surprisingly, when the IL-23R ${ }^{-/-}$allele was crossed into Rag2 ${ }^{-/-}$ mice, we observed exacerbated disease, increased epithelial damage, reduced PSTAT3 in the epithelium, and delayed recovery of IL23R ${ }^{-/-}$Rag2 $^{-/-}$mice. This phenotype was rescued with exogenous IL22-Fc, and epithelial pSTAT3 was restored. Depletion of Thy ${ }^{+}$innate lymphoid cells eliminated the majority of IL-22 production in the colon lamina propria of DSS-treated Rag2 ${ }^{-/-}$mice, suggesting that these are the major IL-23 responsive innate cells in this context. In summary, we provide evidence for opposing consequences of IL-23R on innate and adaptive lymphoid cells in murine colitis.

\section{INTRODUCTION}

Interleukin-23 (IL-23) is a myeloid cell derived, disulfide linked, heterodimeric cytokine comprised of IL-23p19 and IL-12p40, the latter of which is shared with IL-12 and can also exist as a homodimer. ${ }^{1}$ It signals to cells that co-express the proprietary IL-23 receptor (IL-23R) subunit and the shared IL-12R $\beta 1$ chain. ${ }^{2}$ A number of cell types have been described as IL-23R positive and IL-23 responsive, including $\mathrm{CD} 4{ }^{+} \mathrm{T}$ cells of the $\mathrm{T}_{\mathrm{H}} 17$ lineage, $\gamma \delta$ T cells, macrophages, dendritic cells, and innate lymphoid cells. ${ }^{3,4}$ IL-23 signaling involves the cytoplasmic kinases Jak2 and Tyk2, as well as signal transducer and activator of transcription (STAT) $1,3,4$, and $5 .^{2}$ In mice, global deletion of IL-23 or its receptor results in resistance to autoimmune and inflammatory disorders, such as experimental autoimmune encephalomyelitis, ${ }^{5}$ collagen-induced arthritis, ${ }^{6}$ and inflammatory bowel disease (IBD). ${ }^{7,8}$

IBDs such as Crohn's disease and ulcerative colitis are thought to result from a dysregulated immune response to the commensal microflora present in the gut. IL23R has been identified as an important susceptibility gene in Crohn's disease,, 10 and in other immune diseases ${ }^{11,12}$ by several genome-wide association studies. Other components of the IL-23 pathway, namely IL12p40, Jak2, and STAT3, have also been implicated in IBD, ${ }^{10,13}$ further supporting the concept that IL-23 signaling promotes pathogenesis in IBD. However, in spite of the genome-wide association study data, clinical trials with an antibody that neutralizes IL-23 and IL-12 (ustekinumab) have thus far failed to show robust and reproducible efficacy in patients with Crohn's disease, ${ }^{14,15}$ demonstrating that the biology of IL-23 in IBD is incompletely understood.

In murine models of colitis, blockade or deletion of IL-23 ameliorates disease induced by transfer of naive $\mathrm{T}$ cells ${ }^{7,16}$ and Helicobacter hepaticus infection. ${ }^{8}$ The activity of IL-23 has been linked primarily to the maintenance of a $\mathrm{T}_{\mathrm{H}} 17$ response, ${ }^{17,18}$ resulting in increased production of $\mathrm{T}_{\mathrm{H}} 17$-associated cytokines IL-17A, IL-17F, and IL-22. ${ }^{19,20}$ More recently, IL-23 was shown to drive the differentiation of pathogenic $\mathrm{T}_{\mathrm{H}} 17$ cells in the absence of TGF- $\beta .{ }^{21}$ Paradoxically, a predominantly protective role in the context of colitis is ascribed to the major cytokines downstream of IL-23. For example, IL-22 is protective in naive T-cell transfer and chemically induced models of colitis, ${ }^{22}$ as well as colitis caused by infection with Citrobacter rodentium. ${ }^{23}$ IL-17

\footnotetext{
${ }^{1}$ Department of Molecular Biology, Genentech, South San Francisco, California, USA. ²Department of Immunology, Genentech, South San Francisco, California, USA.

${ }^{3}$ Department of Pathology, Genentech, South San Francisco, California, USA. Correspondence: N Ghilardi (ghilardi@gene.com)

Received 16 March 2011; accepted 4 October 2011; published online 16 November 2011. doi:10.1038/mi.2011.54
} 
is either neutral ${ }^{24}$ or protective ${ }^{25}$ in T-cell transfer models, and is protective in chemically induced colitis. ${ }^{26}$ Therefore, eliciting IL-17 and IL-22 production by $\mathrm{T}_{\mathrm{H}} 17$ cells is not likely to be a major mechanism by which IL-23 promotes colitis. Interestingly, a separate mechanism in which IL-23 drives intestinal inflammation by restraining the development of induced regulatory T cells has recently been described. ${ }^{24}$

In contrast to the relatively well-defined consequences of IL-23 stimulation in T cells, the role of the IL-23 pathway in non-T cells remains poorly studied. One previous study demonstrated that innate immune colitis in $\operatorname{Rag} 2^{-1-}$ mice caused by $H$. hepaticus infection is dependent on IL-23, illustrating the relevance of IL-23 signaling in cells other than adaptive lymphocytes. ${ }^{7}$ We decided to examine the consequences of IL-23 signaling in the context of dextran sulfate sodium (DSS)-induced colitis. DSS is thought to directly disrupt the epithelial integrity of the colon, which rapidly leads to inflammation and weight loss. ${ }^{27}$ DSS-induced colitis is commonly considered an innate model, as severe combined immunodeficiency and athymic CD-1 nu/nu mice retain susceptibility. ${ }^{28}$ Here we reveal an unexpected dichotomy in the function of the IL-23 pathway in the adaptive and innate immune compartments.

\section{RESULTS \\ IL-23p19 and IL-23R promote weight loss and colitis in a chemically induced model}

To determine whether IL-23/IL-23R signaling is required for pathogenesis in the DSS model of colitis, we added 3.5\% DSS (wt/vol) to the drinking water for 5 days and subsequently offered normal water. Weight loss is generally reflective of colitis in this model, and consistent with the proposed pathogenic role of IL-23, IL23p19 $9^{-/-}$and IL23R $\mathrm{R}^{-/-}$mice lost significantly less weight compared with wild-type (WT) mice (Figure 1a). Because of the inherent variability of the DSS colitis model, we repeated this experiment three times, and meta-analysis revealed a very significant reduction $(P<0.0001)$ in weight loss in IL23p19-/- or IL23R ${ }^{-/}$mice (Supplementary Figure S1 online). In addition, colons shortened to a lesser degree and histological scores were less severe in IL23p19-/- and IL23R $\mathrm{R}^{-/-}$ mice, relative to WT controls (Figure $\mathbf{1 b}$ and $\mathbf{c}$ ). Histological evaluation of colons deficient in IL-23 signaling revealed decreased leukocytic infiltrate, less crypt loss, and reduced epithelial hyperplasia (Figure 1d).

\section{IL-23 signaling is required for maximal neutrophil infiltration in DSS colitis}

To better characterize the mechanism underlying the protective phenotype in the absence of IL-23p19 or IL-23R, we examined the expression of genes that are known to be regulated by IL-23 in the colons of DSS-treated mice (Figure 2a). As expected, IL-17A and IL-22 expression was increased following DSS treatment, and this increase was dramatically reduced in colons from IL23p $19^{-1-}$ and IL23R $\mathrm{R}^{-1-}$ mice. The Reg3 family of antimicrobial peptides, shown previously to be induced by IL- $22,{ }^{23}$ was also decreased in the absence of IL-23 signaling. In untreated IL23 $\mathrm{R}^{-1-}$ and IL23p19-1- mice, there was a trend toward decreased expression of Reg $3 \beta$ and $\operatorname{Reg} 3 \gamma$, but this was not statistically significant as expression in WT mice was highly variable (Supplementary Figure S2 online). IL-17A and IL-22 synergistically promote expression of inflammatory cytokines and chemokines, leading to the recruitment of neutrophils. ${ }^{29}$ Consistent with reduced IL-17A and IL-22 expression, recruit-

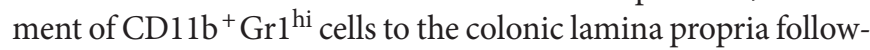
ing DSS exposure was dampened in IL23R $\mathrm{R}^{-/-}$and IL23p19-/mice (Figure $\mathbf{2 b}$ and $\mathbf{c}$ ). The majority of $\mathrm{CD} 11 \mathrm{~b}^{+} \mathrm{Gr} \mathrm{1}^{\mathrm{hi}}$ cells were negative for $\mathrm{F} 4 / 80$; however, there was also a trend toward decreased infiltration of $\mathrm{CD} 11 \mathrm{~b}^{+} \mathrm{Gr} 1^{\mathrm{hi}} \mathrm{F} 4 / 80^{+}$inflammatory monocytes in IL23R $\mathrm{R}^{-/-}$and IL23p19-/- mice (Supplementary Figure $\mathbf{S 3}$ online).

\section{IL-23R is protective in the absence of mature lymphocytes}

Owing to the innate nature of the DSS model, we hypothesized that the pathogenic effects of IL-23 signaling might be mediated by innate immune cells. To confirm the proinflammatory role of non-T or B-cell IL-23R in chemically induced colitis, we crossed the IL23R $\mathrm{R}^{-1-}$ mice with Rag2 ${ }^{-1-}$ mice and again challenged with 3.5\% DSS for 5 days. Surprisingly, however, IL23R $\mathrm{R}^{-1-} \mathrm{Rag} 2^{-1-}$ mice experienced more weight loss than IL23R $\mathrm{R}^{+/+}$Rag2 $2^{-/-}$controls (Figure 3a). At the high dose of $3.5 \%$ DSS, most IL23R $\mathrm{R}^{-1-} \mathrm{Rag}^{-1-}$ mice were moribund at day 8. In order to elicit milder disease and allow for examination of the recovery phase following acute colitis, we administered a lower dose of $2.5 \%$ DSS for 5 days. At this concentration, IL23R $\mathrm{R}^{+/+} \mathrm{Rag} 2^{-/-}$mice lost only about $5 \%$ of their weight and began recovering after day 7 (Figure 3b). In contrast, IL23R $\mathrm{R}^{-/-}$ Rag2 ${ }^{-1-}$ mice lost around $15 \%$ weight and did not recover until after day 10. Consistent with increased weight loss, DSStreated IL23R $\mathrm{R}^{-/-} \mathrm{Rag}^{-/-}$mice had shorter colons (Figure 3c). Histological analysis of colons following DSS treatment revealed increased crypt loss and epithelial damage in IL23R $\mathrm{R}^{-1-} \mathrm{Rag} 2^{-1-}$ mice (Figure 3d and e). IL-22 expression was virtually absent in both DSS-treated and untreated IL23R $\mathrm{R}^{-/-} \mathrm{Rag}^{-/-}$colons (Figure 3f). In addition, expression of IL-17A and the Reg3 proteins were reduced in the absence of IL-23R. Interestingly, Rag2 deficiency lead to very high levels of Reg3 protein expression even in the absence of DSS challenge, and no significant further increase was observed in response to DSS (Figure 3f, compare scale bars with Figure 2a). In mice deficient in both Rag2 and IL-23R, Reg3 levels were dramatically reduced, and again this was independent of DSS challenge (Figure 3f). Furthermore, levels of IL22, Reg $3 \alpha$, and Reg $3 \beta$ are similar when comparing DSS-treated IL23R $\mathrm{R}^{+/+} \mathrm{Rag} 2^{+/+}$and IL23R $\mathrm{R}^{+/+} \mathrm{Rag} 2^{-/-}$colons, but IL-17 is decreased by 5 -fold in IL23R $\mathrm{R}^{+/+} \mathrm{Rag} 2^{-/-}$samples (compare scale bars between Figures $\mathbf{3 f}$ and $\mathbf{2 a}$ ). This observation suggests that adaptive lymphocytes, most likely $\mathrm{CD} 4^{+} \mathrm{T}$ cells, are the predominant source of IL-17A, and is consistent with the observation of Sawa et al. ${ }^{30}$ that IL-22 in the gut is predominantly derived from innate cells rather than $\mathrm{T}$ cells.

In light of the nearly complete absence of IL-17 and IL-22, it was surprising that the leukocytic infiltrate was similar between groups, as indicated by histological evaluation (Figure 3e) and flow cytometry of CD $11 \mathrm{~b}^{+} \mathrm{Gr} 1^{\text {hi }}$ neutrophils in the lamina 

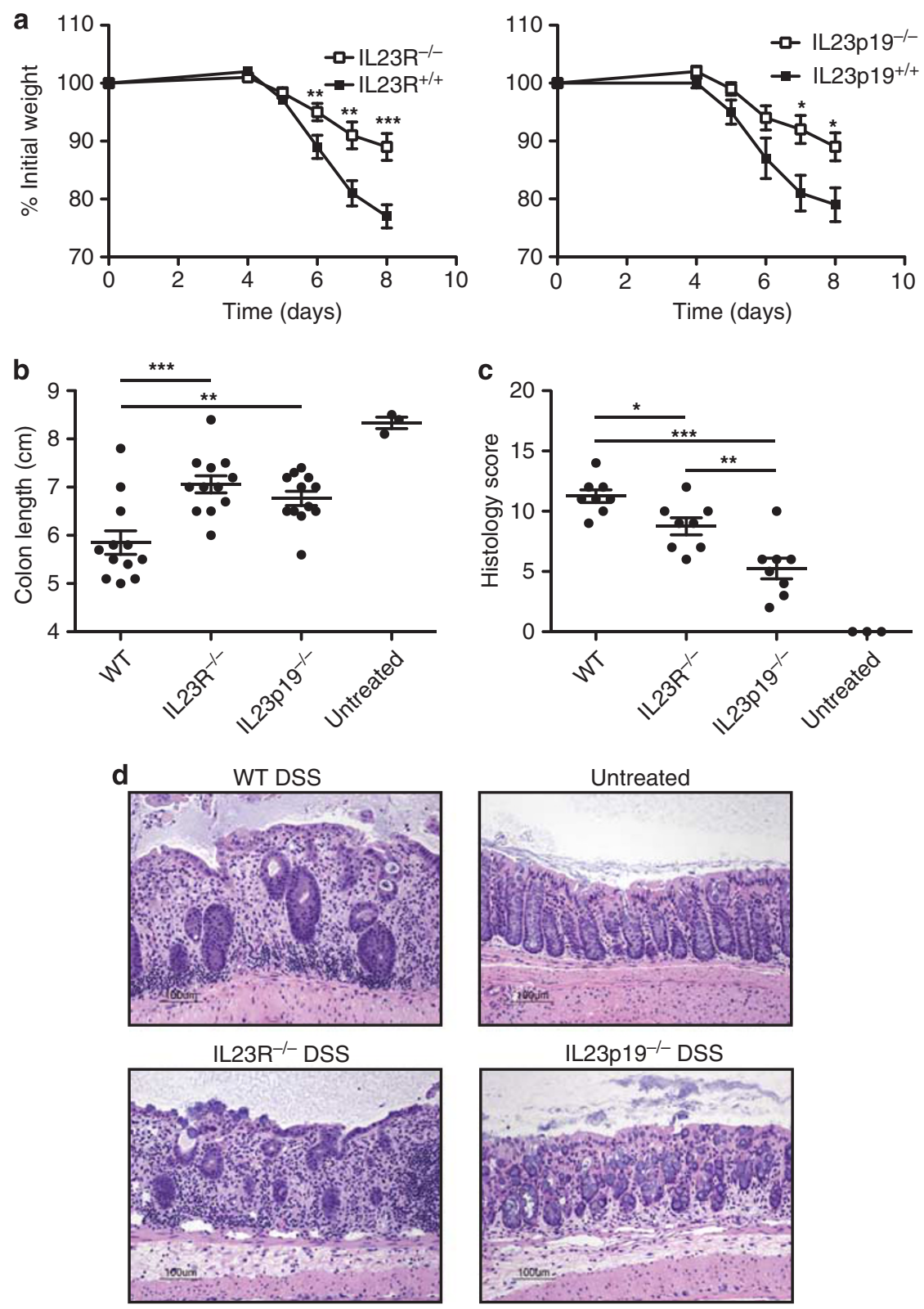

Figure 1 Interleukin (IL)-23 signaling promotes weight loss and histological colitis in dextran sulfate sodium (DSS) model. (a) Wild-type (WT), IL23r ${ }^{-1-}$, and IL23p $19^{-/-}$mice were treated for 5 days with $3.5 \%$ DSS, and weight loss was measured relative to initial weight. ${ }^{*} P<0.05$, ${ }^{* \star} P<0.01$, ${ }^{\star * \star} P<0.001$. (b) Colon lengths from rectum to cecum were determined at day 8 following DSS treatment. (c) Histological scores of colitis and (d) representative hematoxylin and eosin staining for WT, II23r-/-, and II23p19-/- mice are shown. Scale Bar=100 mm. Data are mean \pm s.e.m. Panels $\mathbf{a}$ and $\mathbf{b}$ are representative of three individual experiments, and panels $\mathbf{c}$ and $\mathbf{d}$ are from one experiment.

propria (Figure $3 \mathrm{~g}$ and $\mathbf{h}$ ). The activation of $\mathrm{CD} 11 \mathrm{~b}^{+} \mathrm{Gr} 1^{\text {hi }}$ neutrophils was not suppressed much either, as CD11b intensity was not significantly decreased in cells from IL23R $\mathrm{R}^{-1}$ Rag2 ${ }^{-1-}$ mice (Supplementary Figure S4a online). To investigate the mechanism of neutrophil recruitment in the absence of IL-17 and IL-22, we analyzed the expression of granulocyte-colonystimulating factor, granulocyte-macrophage colony-stimulating factor, CXCL1, CXCL2, and CXCL5 in whole colons, and found these factors to be dramatically more abundant in the combined absence of Rag2 and IL-23R. This result indicates that a compensating, IL-23-independent mechanism takes over to promote neutrophil recruitment when neither IL-23 signaling nor adaptive immune cells are functional (Supplementary Figure S4b online).

\section{IL-22 rescues STAT3 phosphorylation and epithelial repair in IL-23R ${ }^{-/}$- Rag2 $^{-/-}$mice}

Interestingly, the initial kinetics of weight loss following DSS treatment are similar in IL23 $\mathrm{R}^{-/-} \mathrm{Rag} 2^{-/-}$and IL23R $\mathrm{R}^{+/+}$ Rag2 ${ }^{-/-}$mice. However, after the initial phase of disease, the rate of weight loss in IL23 $\mathrm{R}^{+/+} \mathrm{Rag} 2^{-/-}$mice decreases and recovery begins. In contrast, IL23 $\mathrm{R}^{-/-} \mathrm{Rag} 2^{-/-}$mice 
continued to lose weight for 2-3 additional days, suggesting perturbed mucosal repair mechanisms in the absence of IL-23 signaling in innate immune cells (Figure $\mathbf{3 a}$ and $\mathbf{b}$ ). IL-22 has been reported previously to promote mucosal wound healing through STAT3 activation in epithelial cells. ${ }^{31}$ Therefore, we questioned whether lack of IL-22 in IL23R ${ }^{-1-}$ Rag $2^{-1-}$ mice was responsible for the apparent defect in recovery from DSS treatment.
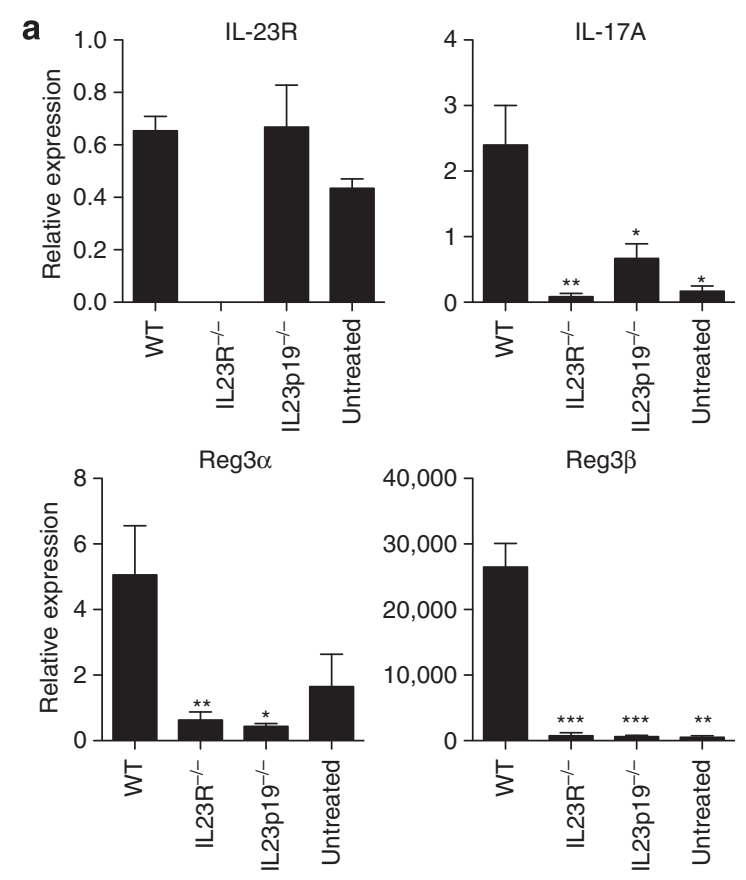
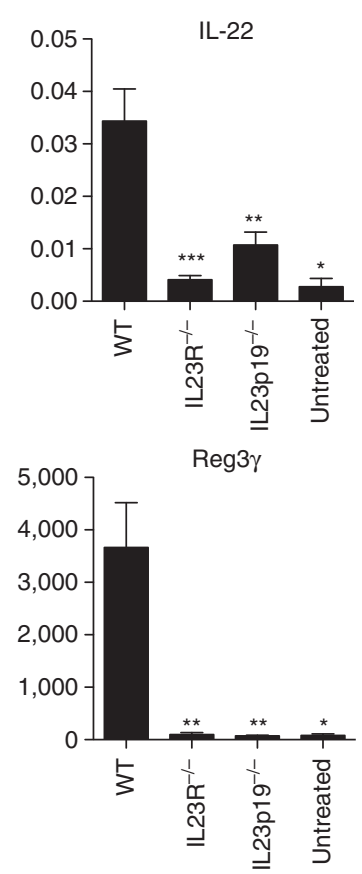

b

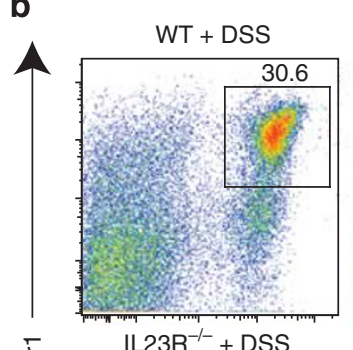

$\stackrel{\bar{c}}{\mathrm{C}}$

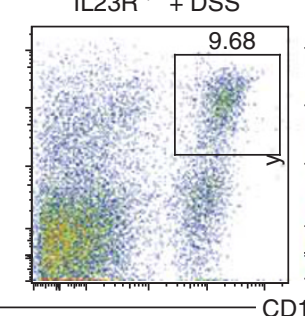

WT untreated

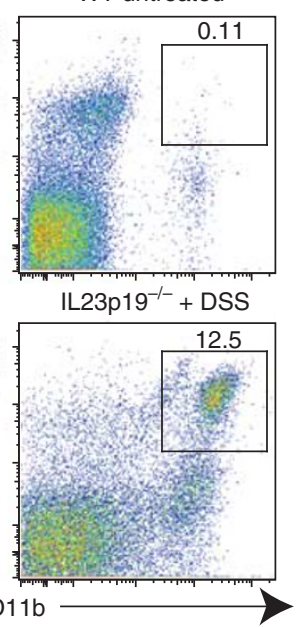

C

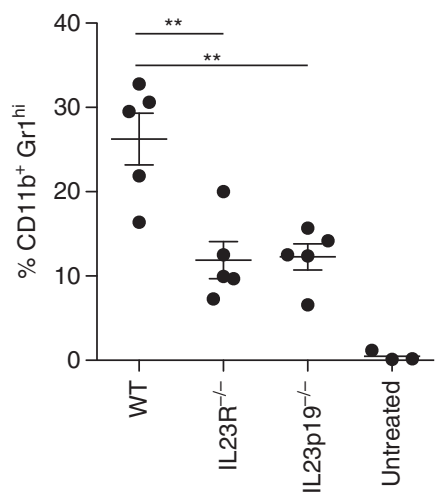

Figure 2 Interleukin (IL)-23 signaling induces expression of Th17 cytokines and promotes neutrophil recruitment following dextran sulfate sodium (DSS) treatment. (a) Relative expression was determined in colons of wild-type (WT), IL23r ${ }^{-/-}$, and IL23p19-/- mice. Arbitrary relative expression units were calculated by division of expression of the gene of interest by Rpl19 mRNA expression and multiplication of the result by 1,000 . ${ }^{\star} P<0.05$, ${ }^{\star \star} P<0.01,{ }^{* \star \star} P<0.001$. (b) Flow cytometry of CD11b ${ }^{+} \mathrm{Gr} 1{ }^{\text {hi }}$ neutrophils in the colonic lamina propria of DSS-treated mice. (c) Quantitative analysis of frequency of $\mathrm{CD} 11 \mathrm{~b}+\mathrm{Gr}{ }^{\mathrm{hi}}$ cells. Data are mean \pm s.e.m., and are representative of two individual experiments.

Figure 3 IL23R ${ }^{-/-}$Rag2 $^{-/-}$mice have increased weight loss and colitis following dextran sulfate sodium (DSS) exposure. IL23R ${ }^{-/-}$Rag2 ${ }^{-/-}$and IL23R $\mathrm{R}^{+/+}$Rag2 $^{-/-}$mice were given $3.5 \%$ (a) or $2.5 \%$ (b) DSS, and weights are plotted relative to weight at day $0 .{ }^{*} P<0.05,{ }^{\star *} P<0.01,{ }^{\star \star *} P<0.001$. Colon lengths (c), colon histology scores (d), representative hematoxylin and eosin staining (e), and colonic expression profiles (f) of mice treated with $3.5 \%$ DSS for 5 days and killed on day 8 . Representative flow cytometry $(\mathbf{g})$ and quantitation of CD $11 b^{+}$Gr $^{\text {hi }}$ neutrophils (h) in the colonic lamina propria of DSS-treated or untreated mice. Data are mean \pm s.e.m., panels a-c are representative of three experiments, panels $\mathbf{d}, \mathbf{e}, \mathbf{g}$, and $\mathbf{h}$ are from a single experiment, and panel $\mathbf{f}$ is from two individual experiments. 
a

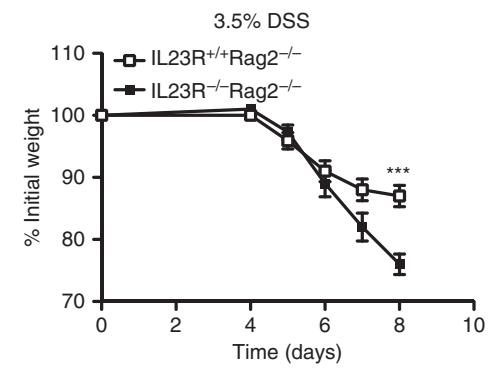

C

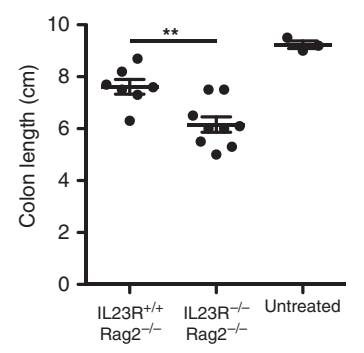

b $2.5 \%$ DSS

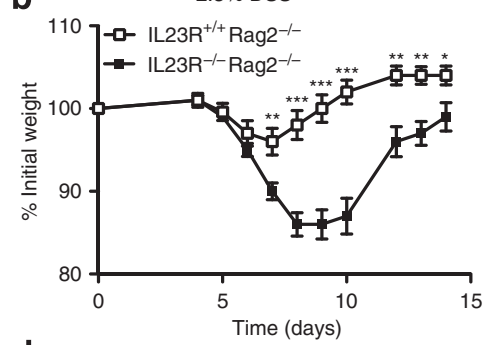

d

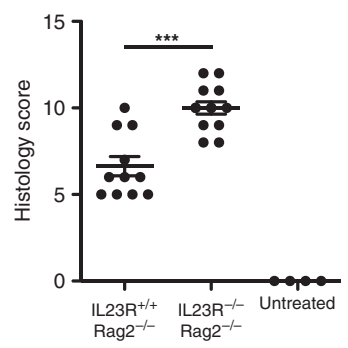

e
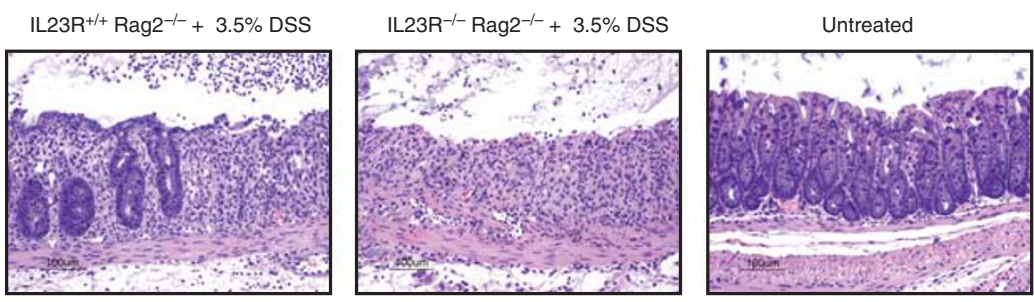

f
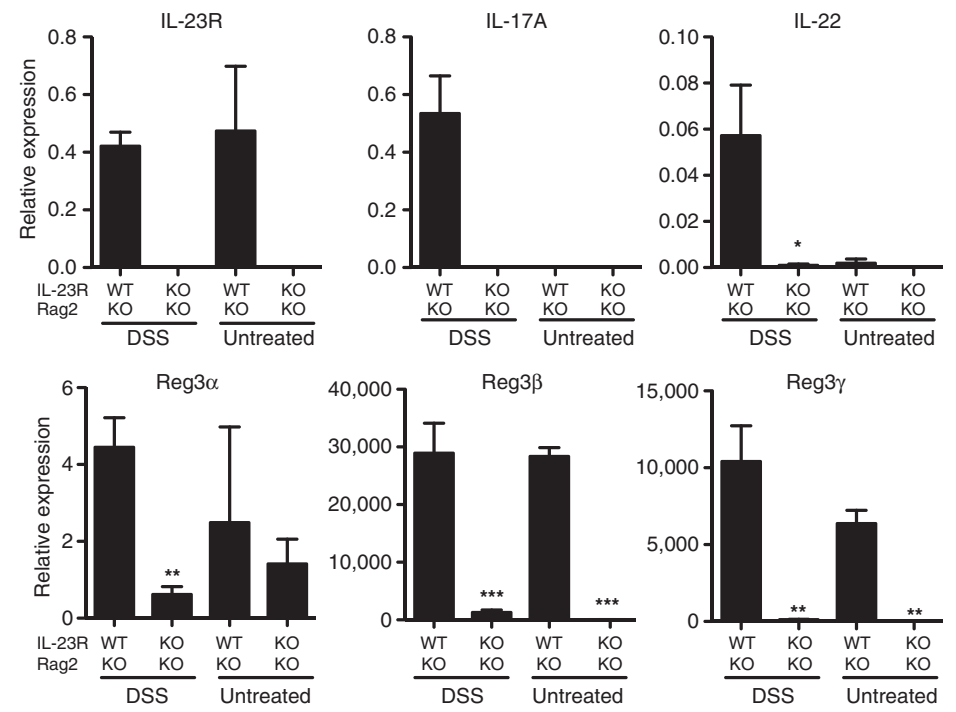

g
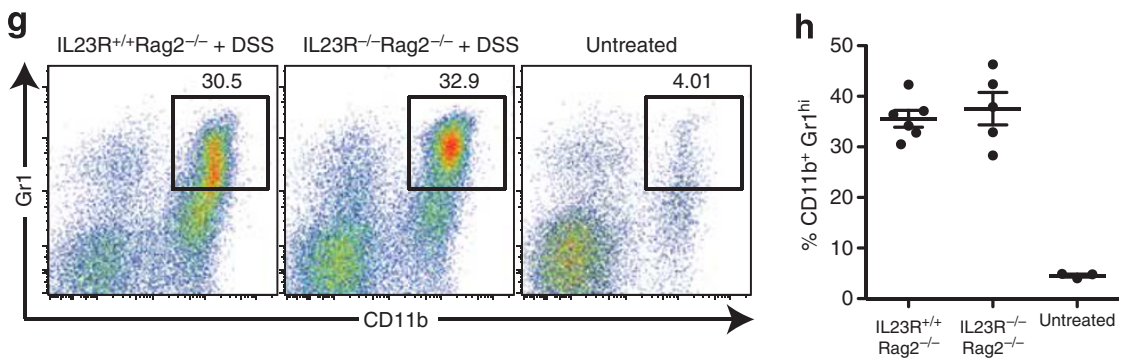
a
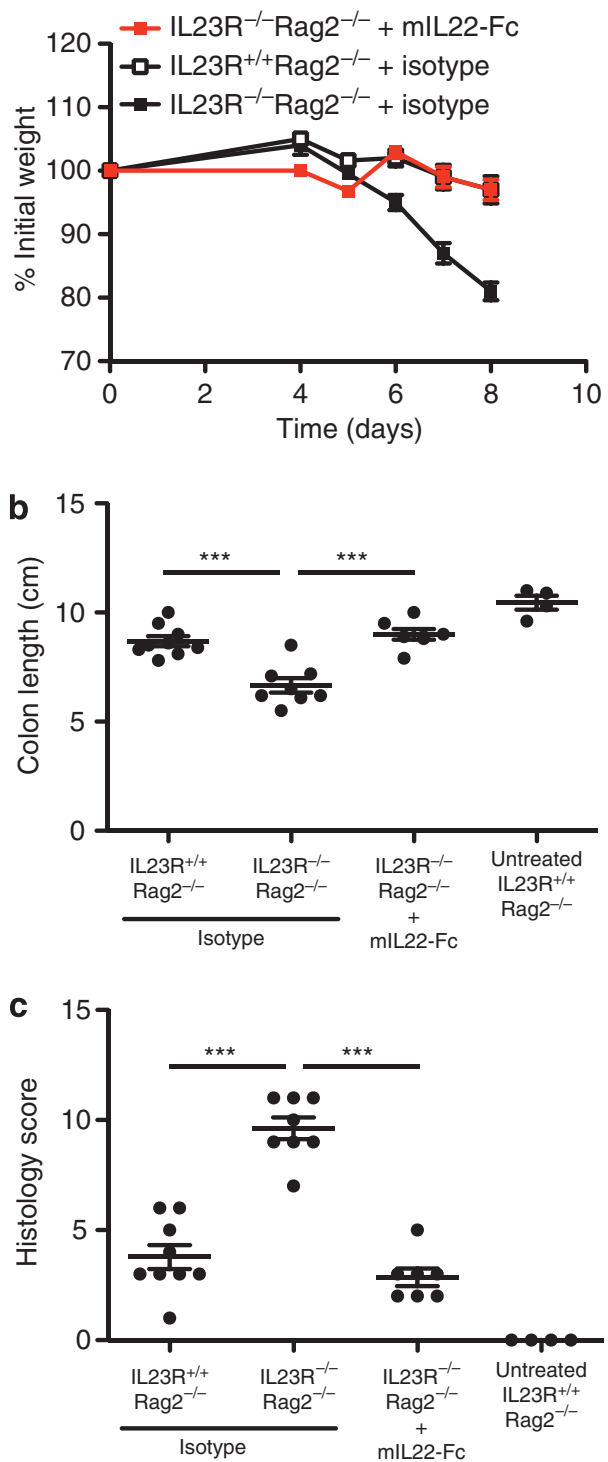

$\mathrm{IL}^{2} 3 \mathrm{R}^{+/+} \mathrm{Rag}^{-/-}$, no DSS

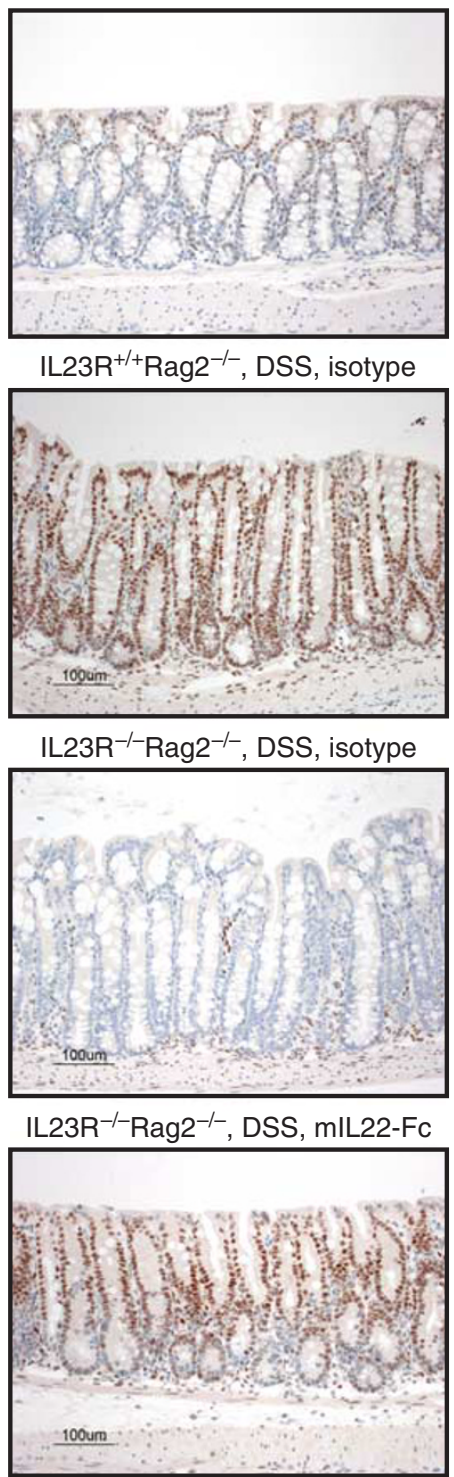

Figure 4 Increased colitis in IL23R ${ }^{-/-}$Rag2 $^{-/-}$mice is rescued by mIL22-Fc through pSTAT3. IL23R ${ }^{+/+}$Rag2 $^{-/-}$and IL23R ${ }^{-/-}$Rag2 ${ }^{-/-}$mice were exposed to $2.5 \%$ dextran sulfate sodium (DSS), and weights are plotted relative to weight at day 0 . Mice were treated with either isotype control or mIL22-Fc (a). Colon lengths (b) and colon histology scores (c) of mice treated with $2.5 \%$ DSS for 5 days and killed on day 8 . Panel $\mathbf{d}$ shows representative images of immunohistochemical staining for pSTAT3. ${ }^{* \star} P<0.001$. Data are mean \pm S.e.m., and are representative of two individual experiments. IL, interleukin; STAT, signal transducer and activator of transcription.

To determine whether IL-22 is sufficient for the protective effects of IL-23 signaling in the DSS model, we attempted to rescue the phenotype by treatment with recombinant IL-22. To maintain IL-22 exposure over time, we used a mIL22-Fc fusion protein because it offers superior pharmacokinetic properties compared to native IL-22. As expected, control treated IL23R $\mathrm{R}^{-/-} \mathrm{Rag}^{-/-}$mice experienced increased weight loss relative to the IL23R $\mathrm{R}^{+/+} \mathrm{Rag} 2^{-/-}$group following 5 days exposure to $2.5 \%$ DSS (Figure 4a). Remarkably, IL23R ${ }^{-/-} \mathrm{Rag}^{-/-}$ mice that received mIL22-Fc experienced minimal weight loss similar to the IL23R $\mathrm{R}^{+/+} \mathrm{Rag} 2^{-/-}$group. IL22-Fc also protected IL23R $\mathrm{R}^{-/-} \mathrm{Rag} 2^{-/-}$mice from colon shortening and histological parameters of colitis (Figure $\mathbf{4 b}$ and $\mathbf{c}$ ). Expression of
Reg3 proteins returned to normal following IL22-Fc treatment (Supplementary Figure S5 online). Immunohistochemical analysis revealed a robust induction of STAT3 phosphorylation in the colon epithelium of IL23R ${ }^{+/+} \mathrm{Rag} 2^{-/-}$mice following DSS treatment (Figure 4d). In contrast, colons from DSS-treated IL23 $\mathrm{R}^{-1-}$ Rag $2^{-1-}$ mice had minimal pSTAT3 staining, which was rescued to normal levels by mIL22-Fc treatment.

\section{Thy $1^{\text {high }} \mathrm{SCA}-1^{+}$innate lymphoid cells are a major source of IL-22 in DSS colitis}

The non-T lymphocyte IL-22 producing cell populations proposed to be responsive to IL-23 include dendritic cells, ${ }^{31}$ natural killer cells, ${ }^{22}$ and most recently Thy $1^{\text {high SCA- }{ }^{+}}$ 
a

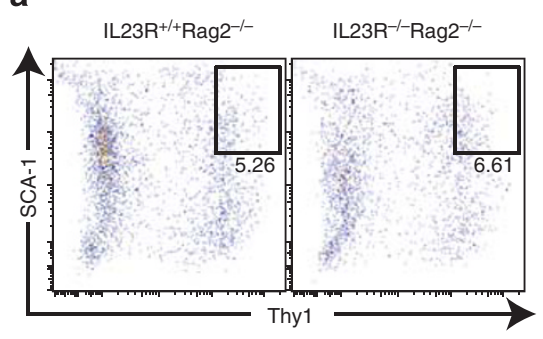

C

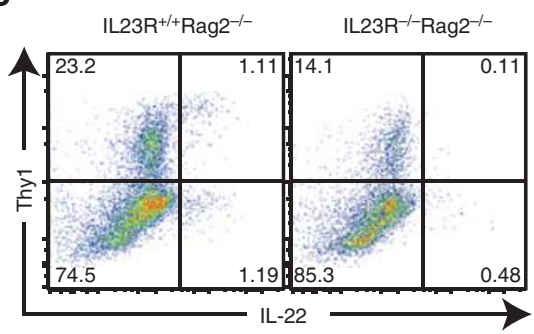

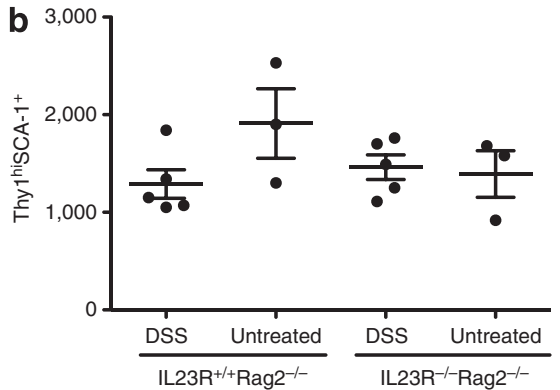

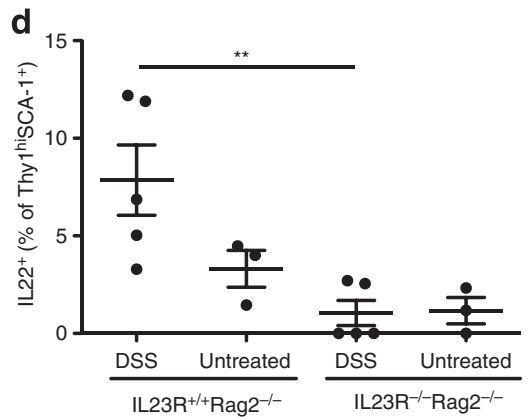

Figure 5 Interleukin (IL)-22 production is increased in Thy $1^{\text {high }}$ SCA-1 ${ }^{+}$cells following dextran sulfate sodium (DSS) treatment of Rag2 ${ }^{-/-}$mice. (a) Representative fluorescence-activated cell sorting (FACS) plots of Thy $1^{\text {high }} \mathrm{SCA}-1^{+}$from the colonic lamina propria (cLP) of mice treated with $2.5 \%$ DSS. Gated on Lin- (Gr1, CD11b, B220, and CD3e negative) cells. (b) Absolute numbers of Lin ${ }^{-}$Thy $1^{\text {high }}{ }^{-}$CA-1 ${ }^{+}$cells in colons of DSS-treated or untreated mice. (c) Representative FACS plots of IL-22 staining in cLP cells from mice treated with 2.5\% DSS. (d) Frequency of IL22 ${ }^{+}$cells (relative to Thy $\left.1^{\text {high }} \mathrm{SCA}-1^{+}\right)$in the colons of DSS-treated and untreated mice. Lamina propria cells were stimulated with phorbol-12-myristate-13-acetate, ionomycin, and brefeldin A, and stained for intracellular IL-22. ${ }^{* \star} P<0.01$. Data are representative of two experiments.

innate lymphoid cells. ${ }^{4}$ In light of the importance of

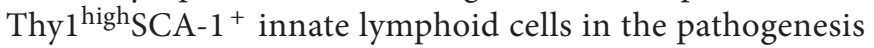
of $H$. hepaticus-induced colitis, we assessed the effect of DSS treatment on the frequency of this cell population. Unlike $H$. hepaticus infection, DSS-induced colitis did not increase the absolute numbers of Thy $1^{\text {high }}$ SCA $-1^{+}$cells in the colonic lamina propria, and levels were normal in IL23 $\mathrm{R}^{-1-} \mathrm{Rag} 2^{-1-}$ mice (Figure 5a and $\mathbf{b}$ ). IL-22 production was detected in Thy $1^{\text {high }}$ cells and also a Thy $1^{\text {low }}$ population (Figure 5c). Intracellular staining revealed that the level of IL-22 in Thy $\mathrm{h}^{\text {high }} \mathrm{SCA}-^{+}$cells was elevated on DSS treatment in IL $23 \mathrm{R}^{+/+}$ Rag2 $2^{-1-}$ mice and was significantly decreased in the absence of IL-23R (Figure 5d). Thy $1^{\text {high } I L 22+}$ cells from DSS-treated mice were characterized to be negative for CD11b, CD11c, and CD4; low for CD117 and NKp46; and positive for SCA-1 and ROR $\gamma$ (Supplementary Figure S6 online).

As DSS exposure induces IL-22 production by Thy $1^{\text {high }}$ SCA$1^{+}$cells, we questioned whether depletion of Thy $1^{+}$cells would reduce IL-22 levels and exacerbate the severity of DSS-induced colitis. As previously reported, ${ }^{4}$ anti-Thy1 treatment efficiently depleted Thy 1 high SCA- $1^{+}$cells in the colons and spleens of DSS-fed Rag2 ${ }^{-1-}$ mice, relative to mice treated with an isotype control antibody (Figure 6a). Thy1 depletion resulted in reduced levels of IL-22 secretion from lamina propria cells cultured either in media or media containing IL-23 (Figure 6b). Furthermore, the expression of IL-17A- and the IL-22-induced proteins Reg $3 \beta$ and Reg $3 \gamma$ were reduced (Figure $6 \mathbf{c}$ ). Thy 1 depletion in mice treated with $2.5 \%$ DSS did not result in altered weight loss, even though there was a significant decrease in epithelial pSTAT3 staining (Supplementary Figure S7 online). However, when the DSS dose was lowered to $2.0 \%$, treatment with $\alpha$ Thyl caused a moderate but significant increase in weight loss (Figure 6d), increased colon shortening (Figure 6e), and more severe histological scores (Figure 6f). Notably, histological evaluation revealed a dramatic loss of epithelial integrity following $\alpha$ Thy 1 treatment, compared with isotype control (Figure 6g). Thus, Thyl depletion resulted in a similar, yet less severe, phenotype as global absence of IL-23R, suggesting that residual IL-22 produced by Thy1-negative cells may still afford some degree of protection. Indeed, reverse transcriptionpolymerase chain reaction analysis of whole colons of Thy1-depleted animals revealed a lesser difference in IL-22 mRNA levels than we expected, based on our analysis of in vitro restimulated cells (Figure $\mathbf{6 b}$ and data not shown).

To corroborate this suspicion, we compared the two conditions in a second model in which both IL-22 and Thy1-positive cells are known to afford protection, namely C. rodentium-induced colitis. ${ }^{32}$ We confirmed the previous observations by Sonnenberg et al., ${ }^{32}$ but found again that Thyl depletion was insufficient to completely phenocopy the severity of disease caused by IL-23R deficiency (Supplementary Figure S8 online).

\section{DISCUSSION}

Blockade of the IL-23 pathway has emerged as a promising therapeutic strategy for several inflammatory diseases, including IBD. ${ }^{33}$ Our data in immunocompetent C57BL/6 mice support 

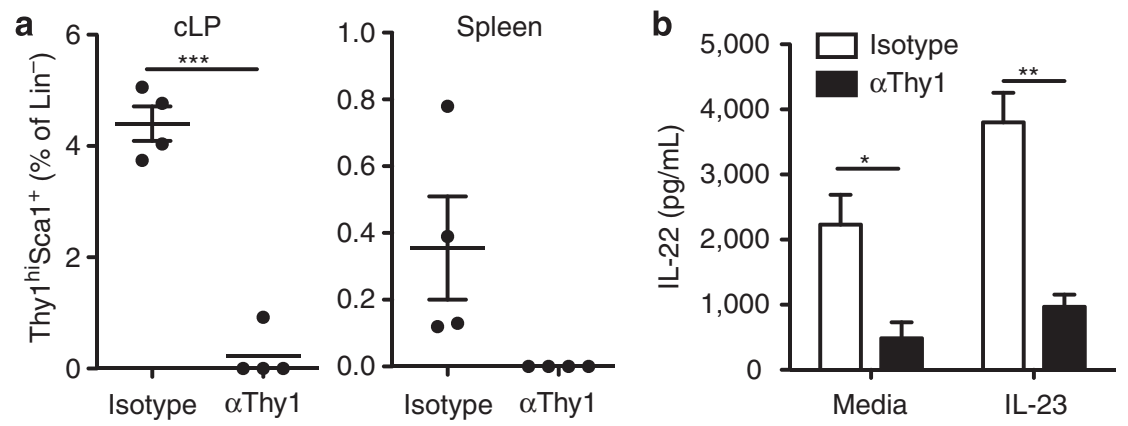

C
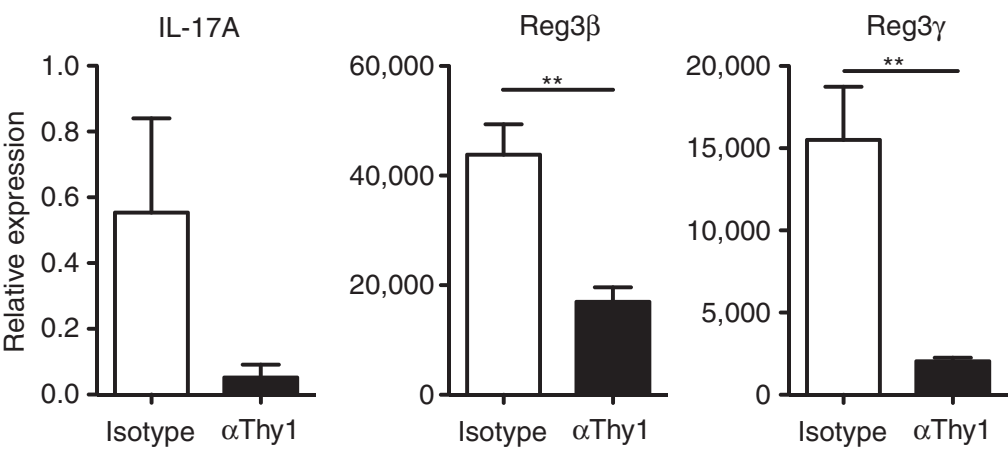

d
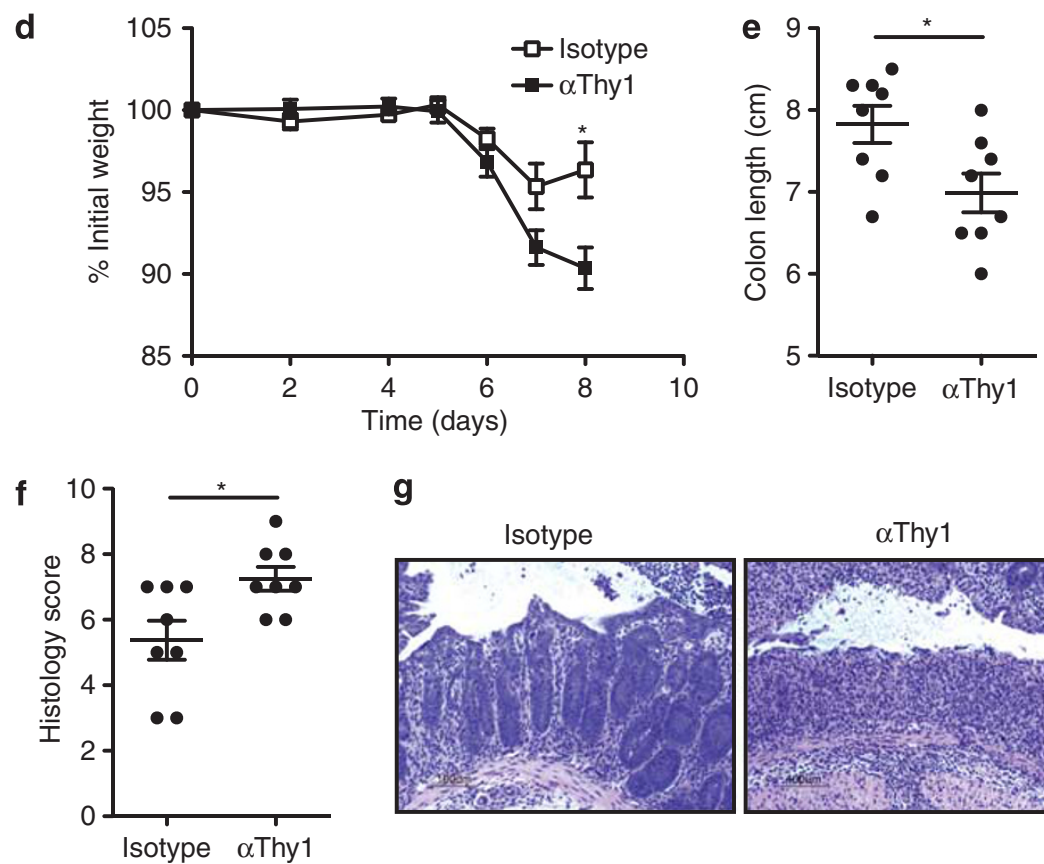

g
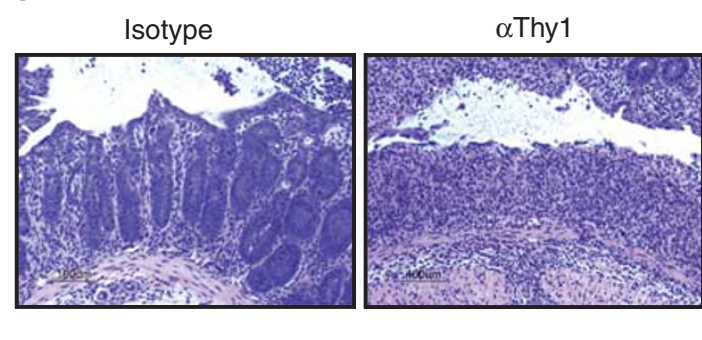

Figure 6 Depletion of Thy $1^{+}$cells in Rag2 ${ }^{-/-}$mice reduces interleukin (IL)-22 production in the lamina propria. (a) Frequency of Thy $1^{\text {high }}$ SCA-1+ cells in colonic lamina propria (cLP) and spleen following depletion with $\alpha$ Thy 1 antibody. All mice were Rag2 ${ }^{-/-}$and were exposed to $2.5 \%$ dextran sulfate sodium (DSS) for 5 days, then normal water for an additional 3 days. (b) Enzyme-linked immunosorbent assay analysis of IL-22 levels from $1 \times 10^{5}$ lamina propria cells cultured overnight in media alone or media containing $10 \mathrm{ng} \mathrm{ml}^{-1} \mathrm{IL}-23$. (c) Reverse transcription-polymerase chain reaction expression analysis of IL17A, Reg3 $\beta$, and Reg3 $\gamma$ in colons of $\alpha$ Thy 1 or isotype control treated mice. (d) Weight loss and (e) colon lengths in $\alpha$ Thy 1 or isotype control treated mice following 5 days exposure to $2.0 \%$ DSS. (f) Histological colitis scores and (g) representative histology of mice treated with $2.0 \%$ DSS. ${ }^{\star} P<0.05,{ }^{* * *} P<0.001$. Data are representative of two experiments.

a therapeutic benefit of IL-23 inhibition and highlight a dominant pathogenic role for the IL-23 pathway in a chemically induced model of colitis. Consistent with the widely accepted colitogenic function of IL-23 signaling, immunocompetent mice deficient in IL-23R or IL-23p19 were protected from
DSS-induced colitis, as determined by weight loss, macroscopic scoring of the colon, and colon histopathology analysis. We observed highly significant protection from DSS-induced weight loss across multiple experiments in both IL23 $\mathrm{R}^{-1-}$ and IL23p $19^{-/-}$mice (Supplementary Figure S1 online). 
Furthermore, our data are consistent with the observation that neutralization of IL-23 (and also IL-12) by aIL12p40-blocking antibody reduces the severity of DSS-induced colitis. ${ }^{34}$ It is therefore surprising that another publication suggested a protective role for IL23p19 in the context of DSS colitis. ${ }^{35}$ Unfortunately, the data presented in that study did not reach statistical significance and were not followed up by any in-depth analysis, and therefore it is not possible to determine the mechanistic underpinnings of the divergent results.

Intriguingly, while receptor and ligand knockouts generally phenocopied each other, we nevertheless found reproducibly that histological scores of IL23p19-/- mice were less severe than those of IL23R $\mathrm{R}^{-1-}$ mice. There were also minor differences in the degree to which expression of IL-17A and IL-22 were affected by ligand or receptor deficiency (Figure 2a). The underlying mechanism for these discrepancies is currently unknown, but several potential explanations can be proposed. First, it remains a formal possibility that IL-23p19 can functionally interact with proteins other than IL-12p40, IL-12R $\beta 1$, and IL-23R. Such subunit promiscuity would not be unprecedented in the IL-12 family of cytokines, as it has been shown that the IL-12p35 subunit can bind both to IL-12p40 and also to a structurally related protein, EBI3 $3{ }^{36}$ An alternative second explanation would be that IL-23p19 and IL-23R deficiency are not functionally equivalent, because they may affect IL-12 signaling in opposing ways. In the case of IL-23R deficiency, IL-23 would still be produced, and would likely accumulate because receptor-mediated endocytosis and degradation could no longer happen in the absence of IL-23R. This free IL- 23 would be predicted to bind to IL- $12 \mathrm{R} \beta 1,{ }^{1}$ thereby sequestering it and making it unavailable to serve as a receptor for IL-12, and hence it would functionally act as an IL-12 antagonist. Conversely, if IL-23p19 is removed, it can no longer compete with IL-12p35 for available p40 subunits, and thus IL-12 production is expected to be increased in the absence of IL-23p19. Third, although both mouse strains are housed in the same room, they have never been in direct contact with each other, and it is thus possible that their gut microflora differs generically, which may lead to subtle differences in intestinal inflammation. Finally, while the IL23R $\mathrm{R}^{-1-}$ strain was generated in C57BL/6 embryonic stem cells, the IL23p19-/- allele was made in the 129 background and backcrossed to C57BL/ 6 for more than 25 generations. ${ }^{37}$ Although the strain is considered fully backcrossed, genes in the immediate vicinity of IL23p19 will still be of 129 origin due to tight genetic linkage.

The cardinal finding of this study is that IL23R $\mathrm{R}^{-1-} \mathrm{Rag} 2^{-1-}$ mice experienced more severe colitis and delayed recovery from mucosal damage. In these mice, production of the downstream cytokines IL-17A and IL-22 is severely compromised, as is the expression of antimicrobial Reg 3 family members. We present several lines of evidence that implicate Thy $1^{\text {high }}$ SCA- $1^{+}$innate lymphoid cells and IL-22 as important mediators of this previously underappreciated protective function of IL-23. First, while the absence of lymphocytes had a dramatic effect on IL-17 production, IL-22 levels were barely affected, suggesting that the majority of IL-22 is produced by an innate cell type in the colon, while IL-17 predominantly comes from lymphocytes. Second, we found epithelial pSTAT3 staining to be severely reduced in IL23R $\mathrm{R}^{-1-} \mathrm{Rag} 2^{-1-}$ mice, which is consistent with the previous observation by Pickert et al. ${ }^{31}$ that IL-22 promotes mucosal healing through elaboration of STAT3 signaling in epithelial cells. It was therefore not surprising that treatment with recombinant IL-22 rescued mice from severe colitis and restored epithelial pSTAT3 staining in IL23R $\mathrm{R}^{-/-} \mathrm{Rag} 2^{-/-}$mice. Finally, depletion of Thy $1^{+}$cells in Rag2 ${ }^{-/}$mice was sufficient to decrease IL-22 production by lamina propria cells ex vivo by $75 \%$ and exacerbate disease. However, the effect of Thy 1 depletion was milder than that of genetic ablation of IL-23R, both in terms of IL-22 reduction as well as disease exacerbation. This difference could not be readily explained by incomplete depletion of Thy1-positive cells, and may therefore reflect the fact that other lamina propria cell types, such as dendritic cells ${ }^{31}$ and natural killer cells, ${ }^{22}$ are also known to produce IL-22 in response to DSS treatment.

In our experiments, we identified noteworthy differences between WT and Rag2 ${ }^{-1-}$ mice. As expected, based on the well-described neutrophil attracting properties of IL-17, 38 IL23 $19^{-1-}$ and IL23R $\mathrm{R}^{-1-}$ mice had reduced levels of neutrophil infiltration following DSS treatment, correlating with decreased colitis and weight loss. Unexpectedly, however, IL-23R deficiency had no effect on neutrophil infiltration in DSS-treated Rag2 ${ }^{-1-}$ mice, despite complete absence of IL-17 expression, suggesting that compensatory mechanisms predominate in the absence of $\mathrm{T}$ and $\mathrm{B}$ lymphocytes. In support of this hypothesis, we found dramatically increased expression of granulocyte-colony-stimulating factor, CXCL1, CXCL2, and CXCL5 in colons in the absence of IL-23R, and while the mechanism leading to this induction remains unknown at this time, it could be due to an increase in bacterial exposure or due to alterations in the commensal microbial profile in the absence of Rag2. Indeed, the basal levels of $\mathrm{CD} 11 \mathrm{~b}^{+} \mathrm{Gr} 1^{\mathrm{hi}}$ cells in the colon lamina propria are elevated in untreated Rag2 $2^{-/-}$mice compared with untreated Rag2 ${ }^{+/+}$mice (Figures $\mathbf{2 b}$ and $\mathbf{3 g}$ ). Therefore, our observations suggest altered leukocyte recruiting mechanisms in the absence of adaptive lymphocytes.

Similarly, the antimicrobial lectins Reg3 $\beta$ and $\operatorname{Reg} 3 \gamma$ have been previously described as being induced directly by IL-22 in colonic epithelial cells. ${ }^{23}$ Indeed, IL-22 and Reg3 proteins are upregulated following DSS treatment of WT immunocompetent mice. However, in colons from untreated IL23R $\mathrm{R}^{+/+} \mathrm{Rag} 2^{-/-}$ mice, the basal levels of Reg $3 \beta$ and Reg $3 \gamma$ were considerably higher than in Rag2 ${ }^{+/+}$mice, yet IL-22 remained low. This heightened basal expression of Reg $3 \beta$ and Reg $3 \gamma$ is dependent on IL-23, because expression was almost undetectable in either untreated or DSS-treated IL23R $\mathrm{R}^{-/-} \mathrm{Rag} 2^{-1-}$ colons. The mechanism leading to increased Reg3 $\beta$ and Reg $3 \gamma$ in mice lacking mature $\mathrm{T}$ and $\mathrm{B}$ cells is currently unknown. However, it is plausible that increased bacterial burden and/or altered composition of the microbial flora in Rag2 ${ }^{-1-}$ mice contribute to elevated Reg3 expression. Aberrant expansion of anaerobic microbes, including segmented filamentous bacteria, has been observed 
in the small intestine of Rag2 $2^{-/-}$mice and was attributed to IgA deficiency. ${ }^{39}$ Further, expression of Reg $3 \beta$ and Reg $3 \gamma$ are induced by increased bacterial exposure ${ }^{40,41}$ apparently through direct recognition of bacterial pathogen-associated molecular patterns (PAMPs) by Paneth cells in a MyD88-dependent manner. ${ }^{40,42}$ As such, the mechanism by which IL-23 promotes Reg3 expression in Rag $2^{-1-}$ mice, in a seemingly IL-22 independent manner, warrants further investigation.

In summary, we describe a previously underappreciated protective effect of the IL-23/IL-22 axis that is partially mediated by Thy-1-positive innate lymphoid cells. As these cells are ROR $\gamma \mathrm{T}$ positive, our observations complement a recent report by Sawa et al. ${ }^{30}$ in which ROR $\gamma \mathrm{T} / \mathrm{Rag} 2$-double-deficient mice also suffered from exacerbated DSS colitis. Furthermore, we previously also described a protective function of the IL-23/IL-22 axis in the C. rodentium model of colitis, in which survival of IL23p19-/mice was reduced upon infection. ${ }^{23}$ We confirmed that IL23R $\mathrm{R}^{-/-}$ Rag2 ${ }^{-1-}$-double-deficient mice are also more susceptible to C. rodentium-induced weight loss (Supplementary Figure S8 online), suggesting that promotion of epithelial repair by IL-23/IL-22 is critical for control of this pathogen by the host. In contrast, IL-23 signaling has pathogenic consequences not only in the context of DSS colitis in immunocompetent C57BL/6 mice as shown here, but also in T-cell-mediated transfer colitis ${ }^{7}$ and, intriguingly, in innate inflammation elicited by $H$. hepaticus infection in the context of Rag1 deficiency. ${ }^{4,7}$ Together, these results illustrate impressively that various inflammatory triggers, host cell types, and signaling pathways can disturb mucosal homeostasis by distinct mechanisms, all of which may result in phenotypically similar inflammation and pathology. In light of this, it becomes clear that the successful development of therapies for human IBD will be critically dependent on our understanding of the inflammatory triggers that lead to inflammation in specific diseases and/or patients.

\section{METHODS}

Mice. IL23p19-/-, 37 IL23R ${ }^{-/-}$(Supplementary Figure S9 online), and IL23R $\mathrm{R}^{-/-} \mathrm{Rag}^{-/-}$mice on C57BL/6 background were bred and maintained under pathogen-free conditions, and tested negative for $H$. hepaticus, rodentium, and typhlonius and positive for segmented filamentous bacteria. All experiments were approved by the Institutional Animal Care and Use Committee of Genentech.

DSS treatment and assessment of colitis. Male mice aged 8-12 weeks were given 2.5 or $3.5 \%$ DSS (36,000-50,000 MW, MP Biomedical, Santa Ana, CA) in drinking water ad libitum as indicated for 5 days. Where indicated, mice were injected intraperitoneally on days 0,3 , and 6 with $0.05 \mathrm{mg}$ $\mathrm{mIL} 22-\mathrm{Fc}$ (mIgG2a) protein or mouse IgG2a as a control. In Thy1-depletion studies, mice were injected intraperitoneally with $1.0 \mathrm{mg}$ rat- $\alpha$ Thy 1 (clone $30 \mathrm{H} 12$, eBioscience, San Diego, CA) or rat IgG2b isotype control on days 0,3 , and 6 . Mice were killed by $\mathrm{CO}_{2}$ asphyxiation and colons were removed, flushed, and the length was measured from rectum to cecum. Tissues were immediately fixed in $10 \%$ buffered formalin, and $4-5 \mu \mathrm{m}$ paraffin-embedded sections were stained with hematoxylin and eosin. Colitis severity was scored in the proximal colon, medial colon, distal colon, and rectum on a scale of $0-5$, with 0 and 5 representing a normal colon and severe colitis, respectively. The scores of four anatomical regions were summed for each mouse to yield a total histological score. Immunohistochemical analysis was performed with pSTAT3 (Tyr705) (Clone D3A7, Cell Signaling Technologies, Danvers, MA).
Flow cytometry. For flow cytometry, lamina propria leukocytes were isolated as described previously ${ }^{23}$ and surface stained with the following antibodies from BD Biosciences (San Diego, CA): CD11b (M1/70), CD3ع (145-2C11), B220 (RA3-6B2), Gr1 (RB6-8C5), Thy1.2 (53-2.1), and SCA-1 (D7). For intracellular cytokine staining, singlecell suspensions from lamina propria were restimulated for $4 \mathrm{~h}$ in RPMI containing $10 \%$ fetal bovine serum with $50 \mathrm{ng} \mathrm{ml}^{-1}$ of phorbol 12 -myristate 13 -acetate and $500 \mathrm{ng} \mathrm{ml}^{-1}$ of ionomycin in the presence of $5 \mu \mathrm{g} \mathrm{ml}^{-1}$ of brefeldin A (Sigma-Aldrich, St Louis, MO). Cells were then fixed in $1 \%$ paraformaldehyde in phosphate-buffered saline, permeabilized with $0.5 \%$ saponin in flow cytometry buffer ( $0.5 \%$ bovine serum albumin in phosphate-buffered saline), and stained intracellularly with PerCP eFluor710-conjugated anti-mouse IL-22 (eBioscience).

Quantitative reverse transcription-polymerase chain reaction. Colons were collected in RNAlater (Ambion, Austin, TX) and homogenized with the Tissuelyser (Qiagen, Hilden, Germany). Total RNA from colons was isolated with the RNeasy kit (Qiagen) using DNA-free DNAse I digestion (Ambion). Taqman one-step RT-PCR (Applied Biosystems, Foster City, CA) was performed with a 7500 Real Time PCR System according to the instructions of the manufacturer (Applied Biosystems). Expression of the gene of interest was normalized to expression of the 'housekeeping' ribosomal protein L19 mRNA. Arbitrary relative expression units were calculated by division of expression of the gene of interest by ribosomal protein L19 mRNA expression and multiplication of the result by 1,000 for ease of display. Primer and probe sequences for each target are as follows or as described previously: ${ }^{23}$ RPL19, forward, 5' -AGCGCATCCTCATGGAGC-3', reverse, $5^{\prime}$-GTCAGCCAGGAGCTTCTTGC-3' , and probe, $5^{\prime}$-GCC TTGTCTGCCTTCAGCTTGTGGA-3' (FAM, TAMRA); mIL-23R, forward, 5' -TGGACTTTTGTCGGGAATGG-3' , reverse, 5' -TCGAAGT GATCTGTTAAATATCCCAA- ${ }^{\prime}$, and probe, $5^{\prime}$-TGGCCATCATGT TGCCGATTTTTTCT-3' (FAM, TAMRA); mIL-17A, forward, 5' -TCGAGAAGATGCTGGTGGGT-3', reverse, 5' -CTCTGTT TAGGCTGCCTGGC-3', and probe, $5^{\prime}$-CTGCACCTGCGTGG CCTCGA-3' (FAM, TAMRA); mIL-17F, forward, 5' -CCCAGGGTC AGGAAGACA-3', reverse, 5' -CCGAAGGACCAGGATTTCT-3', and probe, $5^{\prime}$-CACCATGAACTCCGTCGCCATT-3' (FAM, TAMRA).

IL-22 enzyme-linked immunosorbent assay. Lamina propria cells were cultured in 96 -well round-bottom plates $\left(1 \times 10^{5}\right.$ per well $)$ in complete Iscove's Modified Dulbecco's Medium media with or without $10 \mathrm{ng} \mathrm{ml}^{-1} \mathrm{rmIL}-23$ (R\&D Systems, Minneapolis, MN). After overnight incubation, supernatants were removed. IL-22 enzyme-linked immunosorbent assay was performed as described previously. ${ }^{20}$

SUPPLEMENTARY MATERIAL is linked to the online version of the paper at http://www.nature.com/mi

\section{ACKNOWLEDGMENTS}

We acknowledge the Genentech histology laboratory for specimen processing and staining, Tiffany Yuan and Lee Nguyen for animal husbandry, and Kurt Schroeder for antibody preparation.

\section{DISCLOSURE}

All authors are current or past employees of Genentech. The authors have no additional conflicting financial interests.

(c) 2012 Society for Mucosal Immunology

\section{REFERENCES}

1. Oppmann, B. et al. Novel p19 protein engages IL-12p40 to form a cytokine, IL-23, with biological activities similar as well as distinct from IL-12. Immunity 13, 715-725 (2000).

2. Parham, C. et al. A receptor for the heterodimeric cytokine IL-23 is composed of IL-12Rbeta1 and a novel cytokine receptor subunit, IL-23R. J Immunol 168, 5699-5708 (2002). 
3. Awasthi, A. et al. Cutting edge: IL-23 receptor gfp reporter mice reveal distinct populations of IL-17-producing cells. J. Immunol. 182, 5904-5908 (2009).

4. Buonocore, S. et al. Innate lymphoid cells drive interleukin-23-dependent innate intestinal pathology. Nature 464, 1371-1375 (2010).

5. Cua, D.J. et al. Interleukin-23 rather than interleukin-12 is the critical cytokine for autoimmune inflammation of the brain. Nature 421, 744-748 (2003).

6. Murphy, C.A. et al. Divergent pro- and antiinflammatory roles for IL-23 and IL-12 in joint autoimmune inflammation. J. Exp. Med. 198, 1951-1957 (2003).

7. Hue, S. et al. Interleukin-23 drives innate and T cell-mediated intestinal inflammation. J. Exp. Med. 203, 2473-2483 (2006).

8. Kullberg, M.C. et al. IL-23 plays a key role in Helicobacter hepaticusinduced T cell-dependent colitis. J. Exp. Med. 203, 2485-2494 (2006).

9. Duerr, R.H. et al. A genome-wide association study identifies IL23R as an inflammatory bowel disease gene. Science 314, 1461-1463 (2006).

10. Van Limbergen, J., Wilson, D.C. \& Satsangi, J. The genetics of Crohn's disease. Annu. Rev. Genomics Hum. Genet. 10, 89-116 (2009).

11. Burton, P.R. et al. Association scan of 14,500 nonsynonymous SNPs in four diseases identifies autoimmunity variants. Nat. Genet. 39, 1329-1337 (2007).

12. Cargill, M. et al. A large-scale genetic association study confirms IL12B and leads to the identification of IL23R as psoriasis-risk genes. Am. J. Hum. Genet. 80, 273-290 (2007).

13. Barrett, J.C. et al. Genome-wide association defines more than 30 distinct susceptibility loci for Crohn's disease. Nat. Genet. 40, 955-962 (2008).

14. Mannon, P.J. et al. Anti-interleukin-12 antibody for active Crohn's disease. N. Engl. J. Med. 351, 2069-2079 (2004).

15. Sandborn, W.J. et al. A randomized trial of Ustekinumab, a human interleukin-12/23 monoclonal antibody, in patients with moderate-tosevere Crohn's disease. Gastroenterology 135, 1130-1141 (2008).

16. Elson, C.O. et al. Monoclonal anti-interleukin 23 reverses active colitis in a T cell-mediated model in mice. Gastroenterology 132, 2359-2370 (2007).

17. Mangan, P.R. et al. Transforming growth factor-beta induces development of the $T(H) 17$ lineage. Nature 441, 231-234 (2006).

18. Stritesky, G.L., Yeh, N. \& Kaplan, M.H. IL-23 promotes maintenance but not commitment to the Th17 lineage. J. Immunol. 181, 5948-5955 (2008).

19. Liang, S.C. et al. Interleukin (IL)-22 and IL-17 are coexpressed by Th17 cells and cooperatively enhance expression of antimicrobial peptides. J. Exp. Med. 203, 2271-2279 (2006).

20. Zheng, Y. et al. Interleukin-22, a T(H)17 cytokine, mediates IL-23-induced dermal inflammation and acanthosis. Nature 445, 648-651 (2007).

21. Ghoreschi, K. et al. Generation of pathogenic $T(H) 17$ cells in the absence of TGF-beta signalling. Nature 467, 967-971.

22. Zenewicz, L.A. et al. Innate and adaptive interleukin-22 protects mice from inflammatory bowel disease. Immunity 29, 947-957 (2008).

23. Zheng, Y. et al. Interleukin-22 mediates early host defense against attaching and effacing bacterial pathogens. Nat. Med. 14, 282-289 (2008).
24. Izcue, A. et al. Interleukin-23 restrains regulatory $T$ cell activity to drive T cell-dependent colitis. Immunity 28, 559-570 (2008).

25. O'Connor, W. Jr et al. A protective function for interleukin $17 \mathrm{~A}$ in T cellmediated intestinal inflammation. Nat. Immunol. 10, 603-609 (2009).

26. Yang, X.O. et al. Regulation of inflammatory responses by IL-17F. J. Exp. Med. 205, 1063-1075 (2008).

27. Cooper, H.S., Murthy, S.N., Shah, R.S. \& Sedergran, D.J. Clinicopathologic study of dextran sulfate sodium experimental murine colitis. Lab. Invest. 69, 238-249 (1993).

28. Axelsson, L.G., Landstrom, E., Goldschmidt, T.J., Gronberg, A. \& BylundFellenius, A.C. Dextran sulfate sodium (DSS) induced experimental colitis in immunodeficient mice: effects in CD4(+) -cell depleted, athymic and NK-cell depleted SCID mice. Inflamm. Res. 45, 181-191 (1996).

29. Sonnenberg, G.F. et al. Pathological versus protective functions of IL-22 in airway inflammation are regulated by IL-17A. J. Exp. Med. 207, 1293-1305.

30. Sawa, S. et al. RORyt+ innate lymphoid cells regulate intestinal homeostasis by integrating negative signals from the symbiotic microbiota. Nat. Immunol. 12, 320-326 (2011).

31. Pickert, G. et al. STAT3 links IL-22 signaling in intestinal epithelial cells to mucosal wound healing. J. Exp. Med. 206, 1465-1472 (2009).

32. Sonnenberg, G.F., Monticelli, L.A., Elloso, M.M., Fouser, L.A. \& Artis, D. CD4(+) lymphoid tissue-inducer cells promote innate immunity in the gut. Immunity 34, 122-134 (2011).

33. Melmed, G.Y. \& Targan, S.R. Future biologic targets for IBD: potentials and pitfalls. Nat. Rev. Gastroenterol. Hepatol. 7, 110-117 (2010).

34. Melgar, S. et al. Validation of murine dextran sulfate sodium-induced colitis using four therapeutic agents for human inflammatory bowel disease. Int. Immunopharmacol. 8, 836-844 (2008).

35. Becker, C. et al. Cutting edge: IL-23 cross-regulates IL-12 production in T cell-dependent experimental colitis. J. Immunol. 177, 2760-2764 (2006).

36. Devergne, O., Birkenbach, M. \& Kieff, E. Epstein-Barr virus-induced gene 3 and the p35 subunit of interleukin 12 form a novel heterodimeric hematopoietin. Proc. Natl. Acad. Sci. USA 94, 12041-12046 (1997).

37. Ghilardi, N. et al. Compromised humoral and delayed-type hypersensitivity responses in IL-23-deficient mice. J. Immunol. 172, 2827-2833 (2004).

38. Fossiez, F. et al. T cell interleukin-17 induces stromal cells to produce proinflammatory and hematopoietic cytokines. J. Exp. Med. 183, 2593-2603 (1996).

39. Suzuki, K. et al. Aberrant expansion of segmented filamentous bacteria in IgA-deficient gut. Proc. Natl. Acad. Sci. USA 101, 1981-1986 (2004).

40. Brandl, K., Plitas, G., Schnabl, B., DeMatteo, R.P. \& Pamer, E.G. MyD88mediated signals induce the bactericidal lectin Reglll gamma and protect mice against intestinal Listeria monocytogenes infection. J. Exp. Med. 204, 1891-1900 (2007).

41. Cash, H.L., Whitham, C.V., Behrendt, C.L. \& Hooper, L.V. Symbiotic bacteria direct expression of an intestinal bactericidal lectin. Science $\mathbf{3 1 3}$, 1126-1130 (2006).

42. Vaishnava, S., Behrendt, C.L., Ismail, A.S., Eckmann, L. \& Hooper, L.V. Paneth cells directly sense gut commensals and maintain homeostasis at the intestinal host-microbial interface. Proc. Natl. Acad. Sci. USA 105, 20858-20863 (2008). 